http://jmscr.igmpublication.org/home/ ISSN (e)-2347-176x ISSN (p) 2455-0450 crossref DOI: https://dx.doi.org/10.18535/jmscr/v9i2.12

\author{
Dournal Of Medical Science And Clinical Research \\ IGM Publication \\ An official Publication of IGM Publication
}

\title{
A Study on Osteochondritis Dissecans of the Lateral Process of Talus Involving the Subtalar Joint
}

\author{
Authors \\ Dr Rahmat Ali (Ms (ortho), MCh (ortho) ) ${ }^{1}$, Dr Rajendra Chaudhry (MD Medicine) ${ }^{\mathbf{2}^{*}}$ \\ ${ }^{1}$ Professor and H.O.D (Ortho department), Maharishi Vashishtha Autonomous State Medical College, \\ Basti, UP \\ ${ }^{2}$ Professor and H.O.D (Medicine Department), Maharishi Vashishtha Autonomous State Medical College, \\ Basti, UP \\ *Corresponding Author \\ Dr Rajendra Chaudhry (MD Medicine)
}

Professor and H.O.D (Medicine Department), Maharishi Vashishtha Autonomous State Medical College, Basti, UP., India

\begin{abstract}
Osteochondritis dissecans is a fairly recognized entity affecting the talus dome. We report a case of a 26year-old male with osteochondritis dissecans of lateral process talus which was missed on imaging studies and identified intraoperatively. The patient was treated with curettage of the subchondral cyst at talus, followed by bone grafting and fixation of the osteochondral lesion with a $5 \mathrm{~mm}$ partially threaded cancellous screw. At the 2-year follow-up evaluation, there were no signs of recurrence and the patient resumed his complete activities

Keywords: osteocondritis, OLT, subtalar joint, talus.
\end{abstract}

\section{Background-}

Osteochondritis Dissecans (OCD) of the Talus is one of the uncommon injuries which can easily be missed initially on clinical and radiological examination. They have an incidence of $0.09 \%$ and is the third most common site following knee and elbow. This condition usually affects the patients in their second decade of life after the enchondral ossification fusion, however, it can even manifest at a later stage with male: female of $2: 1$. Albeit, the etiology is less clearly understood, theories such as traumatic, ischemic, genetic, metabolic, or even idiopathic have been proposed in the literature

\section{Case Report}

A 26-year-old male manual labor presented with complains of chronic pain in left ankle joint and difficulty in walking over the preceding 2 years. The pain was insidious in onset, gradually progressive and dull aching in nature, associated with locking episodes, aggravated during strenuous activities and partially relieved with rest. There were no constitutional symptoms associated with the complaints. He had a twisting injury of left ankle following a minor fall 2 year ago which was treated conservatively as an ankle sprain. On examination, he had a mild antalgic gait with no obvious deformity in his left ankle 
and foot. There was tenderness along the lateral aspect of subtalar joint and sinus tarsi. They were no signs of instability and the range of movements in ankle, subtalar, and mid-tarsal joints were within normal limits bilaterally. Anteroposterior and lateral radiographs of the left ankle showed a radiolucent area at the lateral process of the talus (Fig. 1). Magnetic resonance image (MRI) scans showed degenerative changes at the subtalar joint with subchondral cystic lesion measuring $12 \times 10 \mathrm{x}$ $15 \mathrm{~mm}$ within the lateral part of the talus in the posterior subtalar joint. There were 2 osseous loose bodies in the lateral compartment measuring $13 \times 5 \times 12 \mathrm{~mm}$ and $8 \times 4 \mathrm{~mm}$ respectively (Fig. 2). A decision to curette the subchondral cyst was then made since the patient had a failed conservative treatment. The surgery was performed under spinal anesthesia using a direct lateral sinus tarsi approach comprised of a $3-\mathrm{cm}$ incision, starting from the tip of lateral malleolus to the base of fourth metatarsal. The loose bodies in the subcutaneous tissue were removed and a plane between the anterior talo-fibular and calcaneo-fibular ligaments was utilized to expose the subtalar joint capsule. There was an osteochondral fragment measuring $1.2 \times 1.5 \mathrm{~cm}$ with subchondral bone, present on the plantar aspect (subtalar articular surface) of the lateral process of talus, which was missed on the imaging studies. The osteochondral lesion was removed carefully to expose and curette the underlying cyst. The void was filled with the cancellous bone graft harvested from the ipsilateral calcaneum and the fragment was fixed using 4-mm partially threaded cannulated cancellous screw. A below knee slab was applied postoperatively for a period of 2 weeks following which passive mobilization in the form of ankle pumps and tendo-Achillis stretching was started. Partial weight-bearing was allowed at the end of 6 weeks. At the 1-year follow up, the patient related that he had resumed all the activities of daily living along with sports and had no signs of recurrence, and radiographs and computed tomography (CT) scans revealed full bone consolidation and satisfactory articular alignment. The foot and ankle disability score improved from 26.0 preoperatively to 92.3 at the 1 -year follow-up evaluation.

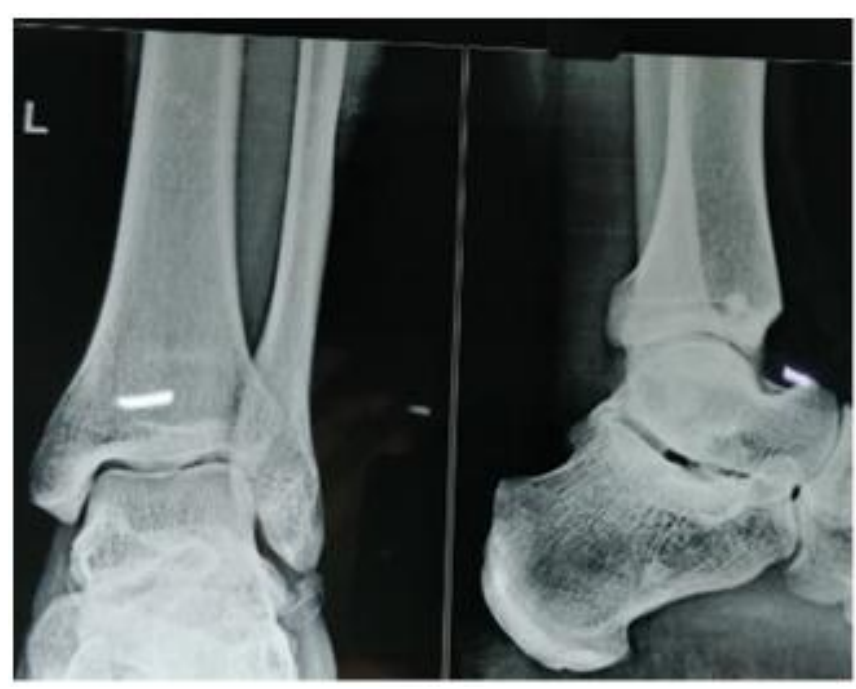

Fig. 1. Anteroposterior (left panel) and lateral (right panel) radiographs of the left ankle showed a radiolucent area at the lateral process of the talus.

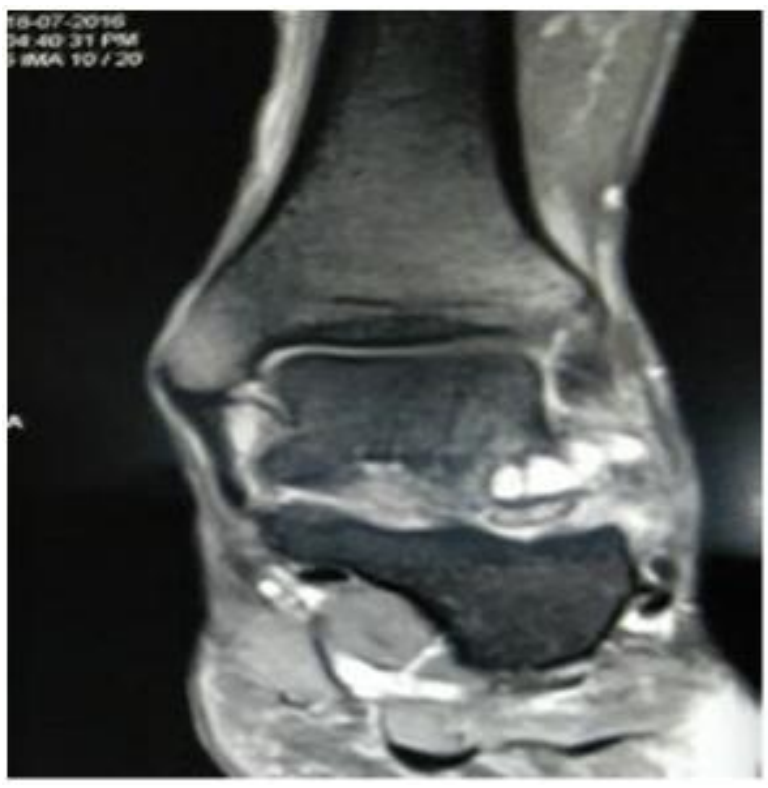

Fig. 2. Magnetic resonance image (MRI) revealing subtalar joint with subchondral cystic lesion measuring $12 \times 10 \times 15 \mathrm{~mm}$ within the lateral part of the talus.

\section{(Pre-Operation MRI)}

\section{Discussion}

Osteochondral lesions are more commonly seen in the lower extremity due to their weight bearing nature. The term osteochondritis dissecans was 
first coined by Franz Konig in 1888 with reference to lose bodies found in the medial femoral condyle. The most common complaints in patients with OCD talus is chronic ankle pain, typically involving the superomedial or superolateral aspect of the talus dome. To the best of our knowledge, this is the first report of OCD involving the lateral talar process in an adult. The initial diagnosis of subchondral cyst affecting the lateral process of the talus was made on the basis of MRI. However, OCD of the lateral process was noted after exposing the subtalar joint. Although, plain radiographs are the first-line of investigation, they can miss the OCD lesions in more than half of the cases initially. The intermittent locking episodes in the present case could be explained due to the loose bodies, just distal to the fibular tip. CT scans provide a better insight for the bony pathologies; however, they have a limited role in detecting the articular cartilage and subchondral lesions where MRI is often considered as gold standard. The primary aim of treatment in the OCD is to achieve revascularization of the involved fragment. While the asymptomatic OCD's involving the subtalar joint can be treated conservatively with nonsteroidal anti-inflammatory drugs, rest and immobilization, symptomatic patients require treatments such as curettage with or without excision, microfracture, antegrade or retrograde drilling, or internal fixation with autologous or allogenous bone graft, autologous chondrocyte implantation or platelet rich plasma. The treatment also depends upon the size, location, grade, presence of subchondral cyst, and extent of joint involvement. There has been a wide variety of treatment options available for OCD affecting the talar dome but paucity of literature still exists for the OCD involving the subtalar joint due to its rare nature. The reason for fixation in the present case was taken on the basis of the size of the fragment which was found to be more than 1.5 $\mathrm{cm}$. In conclusion, an OCD lesion involving the subtalar joint is not a common condition, which mandates a thorough clinical and radiological work-up and prompts management. A differential of OCD should always be kept in mind while treating chronic subtalar joint pathologies. Large fragment can be fixed with a reasonably good outcome.

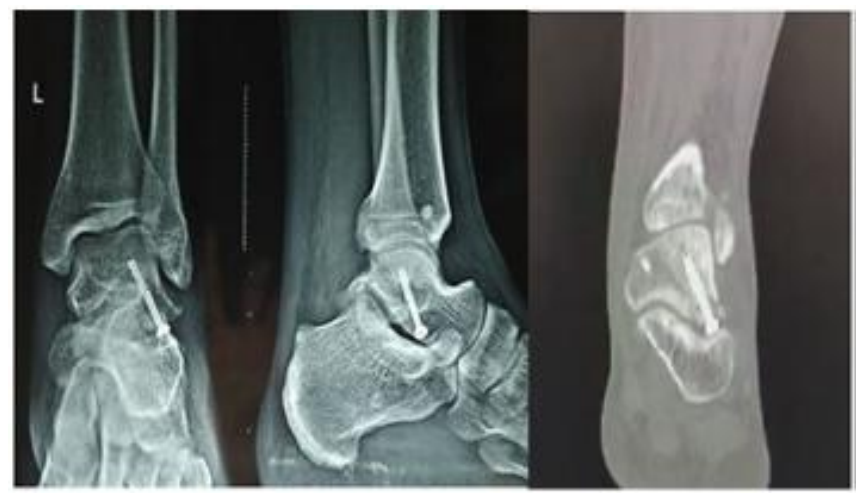

Fig. 3. One-year follow up AP (left panel) and lateral (central panel) radiographs, and CT scans (right panel) revealed full bone consolidation and satisfactory articular alignment. CT, computed tomography.

\section{References}

1. Bruns J, Rosenbach B. Osteochondritis dissecans of the talus. Comparison of results of surgical treatment in adolescents and adults. Arch Orthop Trauma Surg. 1992;112:23-27.

2. Zanon G, Vico GD, Marullo M. Osteochondritis dissecans of the talus. Joints 2014;2:115-123.

3. Higuere J, Laguna R, Peral M, Aranda E, Soleto J. Osteochondritis dissecans of the talus during childhood and adolescence. J Pediatr Orthop. 1998;18:328-332.

4. Lette M, Davidson D, Ahmer A. Osteochondritis dissecans of the talus in children. J Pediatr Orthop. 2003;23:617625.

5. O'Loughlin PF, Heyworth BE. Kennedy JG Current concepts in the diagnosis and treatment of osteochondral lesions of the ankle. Am J Sports Med 2010;38:392-404.

6. Badekas T, Takvorian M. Souras N Treatment principles for osteochondral lesions in foot and ankle. Int Orthop 2013;37:1697-1706. 
7. Vialle R, Madi F, le Pointe HD, Mary P, Maillet M, Damsin JP. Conservative treatment of subtalar osteochondritis dissecans: a case report. Foot Ankle Int. 2007;28:626-629.

8. Nafei A, Saether J, Gelineck J. Osteochondritis dissecans of the calcaneus. Ugeskr Laeger 1990;152:1095.

9. Kadakia AP, Sarkar J. Osteochondritis dissecans of the talus involving the subtalar joint: a case report. J Foot Ankle Surg 2007;46:488-492.

10. Loomer R, Fiher C, Lloyd-Smith R, Sisler $\mathrm{J}$, Cooner T. Osteochondral lesions of the talus. Am J Sports Med 1993;21:13-19.

11. Prado MP, Kennedy JG, Raduan F, Nery C. Diagnosis and treatment of osteochondral lesions of the ankle: current concepts. Rev Bras Ortop. 2016;51:489500.

12. Choi CH, Ogilvie-Harris DJ. Occult osteochondral fractures of the subtalar joint: a review of 10 patients. J Foot Ankle Surg 2002;41:40-43.

13. Boack DH, Manegold S. Peripheral talar fractures. Injury 2004;35:SB23-SB35.

14. Weingi GU, Tanzhu LI, Shi Zhongmin, Mei Guohua, Xue Jianfeng, Zou Jian, Wang Xiaokang, Zhang Haotong, Xu Hongwei. Management of Hepple stage V Osteochondral lesion of the talus with a platelet rich plasma scaffold. Biomed Res Int 2017;2017:6525373.

15. Georgiannos D, Bisbinas I, Badekas A. Osteochondral transplantation of autologous graft for the treatment of osteochondral lesions of the talus: 5 to 7 year follow-up. Knee Surg Sports Traumatol Arthroscop 2016;24:37223729.

16. Giza E, Delman C, Coetzee JC, Schon LC. Arthroscopic treatment of talus osteochondral lesionswith particulated juvenile allograft cartilage. Foot Ankle Int 2014;35:1087-1094. 